\title{
A Novel Elbow Pneumatic Muscle Actuator for Exoskeleton Arm in Post-Stroke Rehabilitation
}

\author{
Mae Irshaidat, Majeed Soufian, Alaa Al-Ibadi and Samia Nefti-Meziani
}

\begin{abstract}
Rehabilitation interventions play a vital role especially in most post-stroke care. Many rehabilitation robotic devices have been designed and developed to assist many individuals suffering from stroke or similar disabling illness and living with reduced mobility of the arms, hands and other joint. However, these devices remain unmanageable to use by the patients alone not only because they are cumbersome to use but also due to their weights, fix and non-portable characteristics, and so on. Here for elbow joint rehabilitation, we investigate and propose a novel exoskeleton soft robotic arm, which is wearable, lightweight and portable so that it would allow patients to perform repetitive motion therapy more often with a greater intensity in their homes and relevant to their daily activities. The proposed arm consists of various novel pneumatic Muscle Actuators (pMA) capable of bending in contrary to traditional pMA. Analysis, design, integration and characterisation of the proposed arm are presented and geometrical and numerical models are obtained. Various experiments revealed its behaviour and the relationship among pressure, length, force, and bending angle in different setups such as isotonic and isometric. The nonlinear, time varying and intractable dynamic of the constructed prototype demanded the design and development of an appropriate closed loop controller for adhering to target rehabilitation profiles. As proof of the concept a Model Reference Adaptive Control (MRAC) was designed and results were presented. By achieving design objectives, this study shows that the proposed portable exoskeleton has the ability to offer more effective intense rehabilitation therapies at home without the need to therapists and with a lower cost.
\end{abstract}

Keywords - upper extremity; limbs; rehabilitation; therapy; soft robots; movement; motion; robot; elbow rehabilitation

\section{INTRODUCTION}

Stroke is one of the major health problems as it is considered to be leading causes of disability in the UK, leaving people with limited ability to perform activities of

Mae Irshaidat is with the Autonomous Systems and Advanced Robotics Centre, School of Computing, Science and Engineering, University of Salford, Manchester, United Kingdom. (e-mail: m.m.irshaidat@edu.salford.ac.uk)

Majeed Soufian is with the Autonomous Systems and Advanced Robotics Centre, School of Computing, Science and Engineering, University of Salford, Manchester, United Kingdom. (e-mail: m.soufian@salford.ac.uk)

Alaa Al-Ibadi is with the Autonomous Systems and Advanced Robotics Centre, School of Computing, Science and Engineering, University of Salford, Manchester, United Kingdom. And with the Computer Engineering Department, University of Basrah, 61004, Basrah, Iraq (e-mail:a.f.a.alibadi@edu.salford.ac.uk)

Samia Nefti-Meziani is with the Autonomous Systems and Advanced Robotics Centre, School of Computing, Science and Engineering, University of Salford, Manchester, United Kingdom. (e-mail: s.neftimeziani@salford.ac.uk) daily living (ADL). Age is playing a significant factor in increasing the chance of suffering a stroke as the probability of suffering a stroke increases proportionally to age [1].

Concerning the upper and lower limbs, damaged arm and leg function may cause serious limitations in activities of ADL for the majority of stroke patients [2]. Directly after stroke, upper and lower limbs weakness is the most common result, occurring in $77 \%$ of patients with a first-ever stroke longitudinal follow-up studies revealed that $60 \%$ of stroke patients regain very little dexterity after six months [3] Providing stroke survivors with the required treatment that they need to recover and regain motor function is a remarkable challenge for physiotherapists. Not only is the process physically difficult for the therapist, but also it requires time and manpower that health care system struggles to provide. Unfortunately, it means that patients are usually not able to get as much therapy as needed. Thus, the need of rehabilitation robotic device was required to assist therapists with assessing stroke patients and providing the necessary therapy. One of the main goals of robotic therapy units is to increase the efficiency of the recovery process. Rehabilitation Robotic devices must be able to perform specific movements to stimulate motor plasticity (flexibility) in the patient's limbs to provide therapy at the same level as a therapist, which will result in improving recovery and minimising functional shortages in upper and lower limbs for the patient. Many assistive systems for limbs rehabilitation have been developed several types of limb rehabilitation. These systems implement distinctive mechanical structures, designs, actuators, techniques, control systems and rehabilitation approaches, as well as various techniques to ensure the reliability and strength of the systems when compared to others. The fast development of rehabilitation robotics over the last ten years is working toward fully regaining or improving the movement of affected limb functions and helping patients achieve a better quality of life [4]. Between the late 1980's and early 1990's engineers started exploring the opened opportunities to utilise robotic devices in rehabilitation therapy [5]. The first robotic devices for rehabilitation such as Lido and Biodex machines that considered active dynamometers robots were developed in late 1970 's.

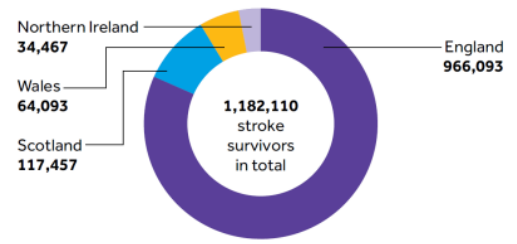

Figure 1. Number of Stroke Survivors in the UK for the year 2016/2017 
These robotic devices aimed to provide activities that allow the patient to exercise and practice independently, moving the limb, and measure the movement performance.

Several rigid robotic devices are able to support more than one joint at the same time, to assist both shoulder and elbow, such as MIT-Manus (Massachusetts Institute of Technology Manus), MIME (Mirror Image Movement Enabler) [6], Assisted Rehabilitation and Measurement Guide (ARM) guide, and GENTLEs [7]. The robotic therapy target for these robots is the same; active assist exercise. Where active refers to the patient's ability to be active and engaged. The assist refers to the therapist assistance provided to patients as needed [8]. Moreover, these robots are easy to adjust with the human upper limb length, which make it simpler and easier to manufacture. However, determining the movement of the upper limb is not easy with only one interface (patients hand). End-effector based robots cannot control the torque at particular joint, in addition, the range of motion (ROM) is limited. as a result, a limited set of rehabilitation exercises can be generated by this rehabilitation robotics.

On the other hand, one of the most important factors that should be taken into consideration while designing a rehabilitation robotic device is the safety in the interaction with patients. To provide safety one of two possibilities can be implemented during the interaction with patients:

- Provide security by controlling the actuators.

- Use safe actuators to absorb unwanted forces produced by human-robot interaction tasks.

Pneumatic actuators are considered one of the safe actuators, where pressurised air is used as main energy source. The compressibility of air helps these actuators to absorb unwanted force. Moreover, pneumatic actuators have a high force/weight ratio, which make robots much lighter. However, the main drawbacks are the high nonlinearity nature due to the air compression and elastic viscos. In addition to the high flow of the volume that used to fulfil the cylinder. Pneumatic muscles can mimic the human muscles by applying air in the chambers. There are several robotic devices for upper limb rehabilitation driven by pneumatic actuators such as SRE, RUPERT, PNEX-WERX, and iPam.

Robotic devices used for upper limb rehabilitation differ from each other by several factors such as: the DOF, joint movements they support, main control inputs, mechanical design and structure, and type of assistance. Kiguchi [9], MARIONET-Suzler [10], Rosen [11], and Song [12], all are robotic devices that assisting elbow movements. However, some of them are classified end-effector based such as MARIONET-Suzler and Song, and to exoskeleton based as in Rosen and Kiguchi. The inputs signal vary from one device to another, in the mentioned devices above the input signal is sEMG except in MARIONET-Suzler as it is the joint angle. In addition, they all share the same degree of freedom 1DOF. Kiguchi is utilised to support the shoulder joint with 2DOF [13].

REHAROB is an industrial rehabilitation robotic that is used for the upper limb motion therapy for disabled, in other words, it is used for physical therapy. It was developed to support the upper limb joints, shoulder and elbow.
REHAROB can also be used in the assessment, as it quantifies the patient's range of motion (ROM) [14].

A major problem with current rehabilitation robotic devices is that the upper and lower limbs mobility is hardly replicated. Many hours are spent in stroke rehabilitation in order to reduce the effects of injuries and improve mobility to enable the stroke patients to perform some activities of daily living.

The aim of this study is to design a wearable robot allowing patients to wear them at home, for low cost, ease, low weight to power ration, strong, and safe use. Soft Robots -pneumatic Muscle actuators-; extensors are used in the design, which are less harmful and human friendly. The behaviour of extensors such as pressure, length, bending angle, and force are illustrated. Moreover, the device is distinguished by its ability to fit human's upper limb because it's adjustable, ease use, and simplicity. Basic controller is used as well to control the soft arm to perform elbow's flexion extension movements.

\section{Pneumatic Muscle ACtuator}

Pneumatic Muscle Actuator (pMA) was used in the soft arm design that is also known as McKibben muscle. McKibben muscle is chosen over the other actuators that have been already mentioned in the literature review due to its capability to produce force required to actuate the muscle. PAM consists of stretchable inner tube (bladder); butyl rubber was used in the design, inserted into a braided mesh (sleeve) made of Polyethylene terephthalate (PET) that belongs to polyester family. In addition, other material such as nylon can be used for the braided sleeve. Plastic cable ties were devised to attach and seal the bladder and braided mesh onto stainless steel fittings (end caps). One of the end caps is fabricated so that air fitting can be attached to allow pressurised air inlet. Fig. 2 shows the pMA build up.

The pMA can be either contraction or extension muscle and the braided angle $\theta$ defines the type of actuator $[15,16]$. Since, the bending is the required behaviour, the self-bending contraction actuator (SBCA) by Al-Ibadi, et al. [17] and the extensor bending pneumatic artificial muscles (EBPAMs) by Al-Fahaam, et al. [18] are used.

\section{A Novel ElBow PMA EXOSKELETON ARM (EPMAE)}

The proposed prototype with $1 \mathrm{DOF}$ is devised to rehab the elbow joint, the prototype consists of two bending extensor pMAs and one bending contractor pMA. The main requirements for the soft arm are:

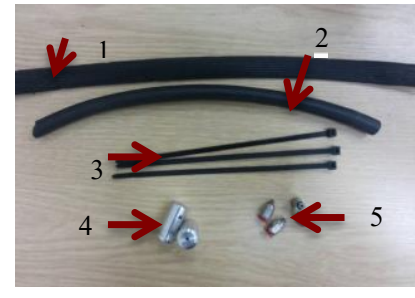

(a)

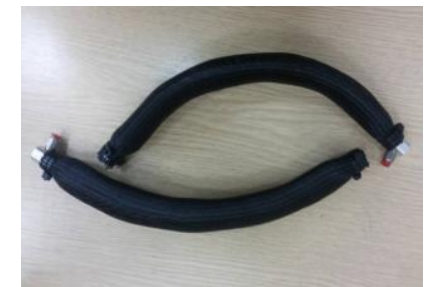

(b)
Figure 2. a) pMA components: 1. Braided mesh, 2. Inner tube, 3. Cable ties, 4. End caps, 5. Air inlet, b) pMA Build-up 


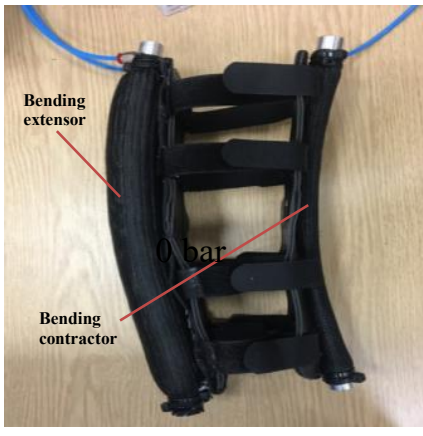

(a)

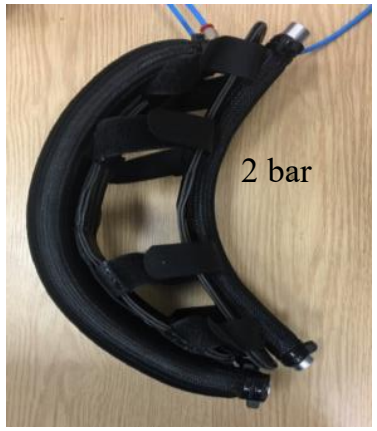

(b)
Figure 3. The proposed soft arm (EpMAE) shown roughly in one to ten reference scale: a) when the soft arm is unpressurised, b) when it is pressurised by approximately 2 bar.

Portable and lightweight

- High force to weight ratio

- Low/no maintenance

- Low cost

- Safe as it is in direct contact with the human

- Compliance

\section{A. EpMAE Construction}

The construction of the proposed prototype depends on the arthrometric data, of the human body as the average mass for the forearm for both genders is $1.5 \mathrm{~kg}$ [19]. The (EpMAE) is distinguished by being soft not only because of the actuators but also the other parts of the exoskeleton arm are bendable and soft. In addition, it does not have any discrete joints; to avoid aligning the exoskeleton arm to the user's arm that might lead to injuries if it does not attach correctly resulting in more adjustments to fit any person.

The bending pMAs are sewn onto the adjustable elbow support as shown in Fig. 3; three bending pMAs are used to increse the genertaed force to flex and extend the arm when the pMAs are pressurised; two bending extensors, on the distal side of the arm, and one bending contractor pMAs, on the oposite side. EpMAE has a total weight of $0.35 \mathrm{~kg}$ and the same construction as in the traditional pMAs and the bending pMAs are implemented.

\section{B. Experimental Inspection for EpMAE}

Few experiments are conducted in order to validate the effectiveness of the EpMAE especially for the case that the prototype is unloaded and loaded with $1.5 \mathrm{~kg}$ weight. It was also of interest to find a relationship between the bending angle of the prototype and the pressure applied. Therefore, an isometric configuration is set to find the relationship of the generated force at different pressure levels. Fig. 4. Illustrate the designed system setup to achieve this isometric configuration and to measure the generated force accordingly.

As shown in Fig. 4a and Fig. 4b, the experiment is designed in a such a way that top of soft arm is fixed at top and free at it end where the load is attached. The pressurised air gradually pumped into the muscle at different pressures varying from 0 to 5 bar with different loads attached to the end of the muscle.

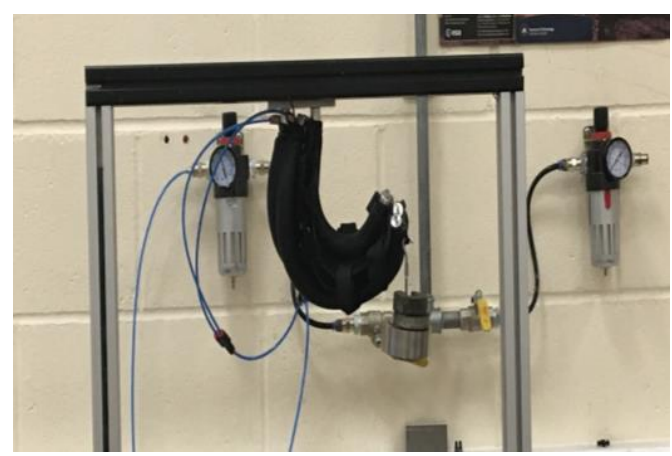

(a)

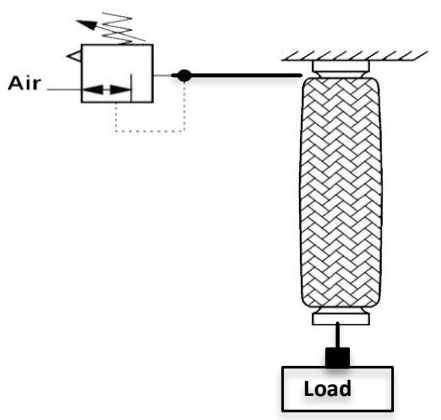

(b)

Figure 4. System Setup to measure the generated force: a) A photo of the prototype setup to find the force of the at different pressure level, showing when the load is $4 \mathrm{~kg}$ at 4 bar, b) Block diagram used for free body diagram to abstract system setup for measuring the generated force.

Fig. 5a. represents a plot of the obtained bending angle of the proposed prototype versus the applied pressure using the experimental model when the prototype EpMAE is free and unloaded. While the measured bending angles of the prototype versus the applied pressure when it is loaded with $1.5 \mathrm{~kg}$ weight are shown in Fig.5b. The results of this two graphs show a nonlinear relationship between bending angle and the applied pressure, which changes dramatically its shape when the proposed prototype is loaded.

In addition, the corresponding force generated from the mechanical motion of the muscle was measured experimentally to investigate the relationship between the pressure and force. The generated forces of the proposed prototype versus the applied pressure illustrated approximately a linear relationship between them and are presented in Fig. 6.

\section{CONTROL DEVElopment}

Here the basic concepts and requirements for developing and design of an appropriate control system for EpMAE are presented. The primary aim of the control system is to make EpMAE suitable for rehabilitation purposes by satisfying its relevant requirements. This is a challenging task especially because of distributed parameter nature of the proposed soft arm (EpMAE) and its nonlinear dynamic that have been discussed in previous sections put strong limitation on applying conventional control techniques. To achieve this, in first place, an appropriate control strategy was chosen and control law was then synthesised based on dynamic characteristics of the constructed EpMAE prototype. 


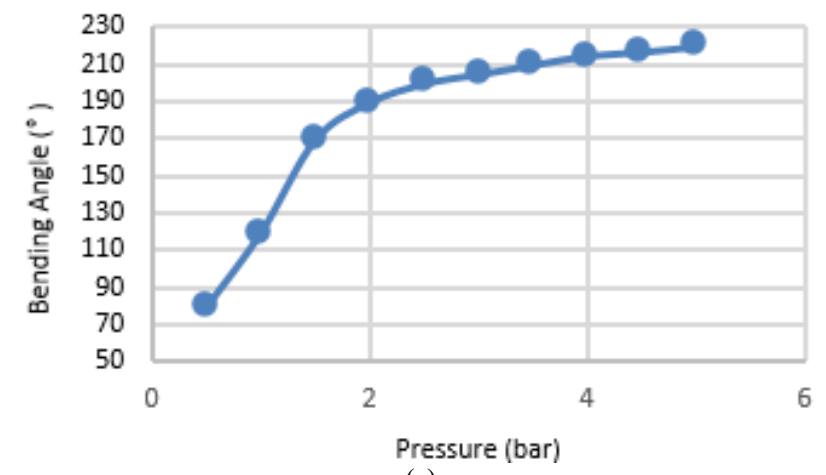

(a)

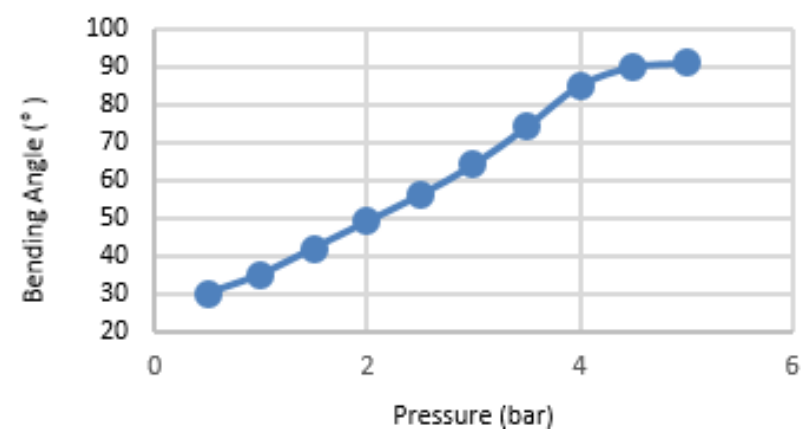

(b)

Figure 5. The observed bending angles for the bending prototype at different pressure levels; (a) shows the nonlinear relationship between the angle in degree and the pressure in bar when the prototype EpMAE is free and unloaded, (b) when the prototype is loaded with $1.5 \mathrm{~kg}$.

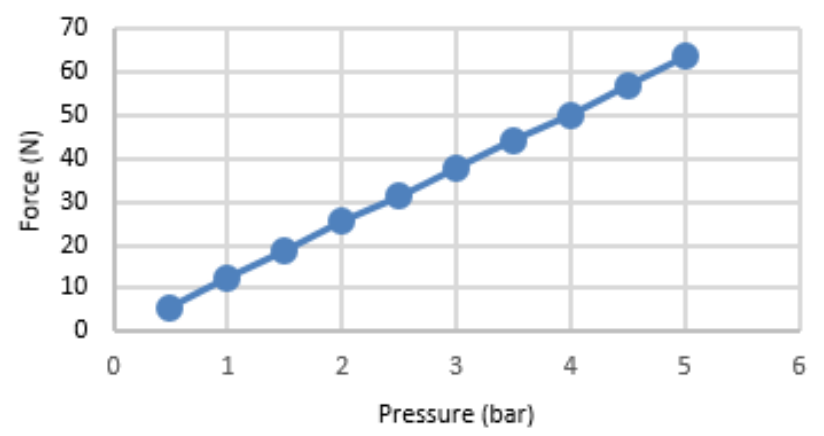

Figure 6. $\boldsymbol{A}$ relationship between the experimental model for the prototype force vs. pressure level.

\section{A.Pneumatic System Setup}

As mentioned earlier, the constructed prototype of elbow arm is made of 3 bending pMAs with a range of motion, which expected to vary from $0^{\circ}$ to $180^{\circ}$ ideally. In the form of pressure pulse, the compressed air flow into the muscles through the solenoid inlet valve so that the resulting pressure $\mathrm{P}$ in the muscles is manipulated variable with different values from about 0 to 5 bar. To control the bending angle of the EpMAE prototype (angle of rotation $\operatorname{arm} \theta$ ) the pressure must be controlled via a solenoid valve through a balance of both tensile forces depending on load and existing air pressure in each muscles of the actuator arm.

Accordingly a pneumatic system is designed and set up to supply the air flow required for the operation of the
EpMAE prototype. The schematic overview of the complete pneumatic system is illustrated in Fig. 7, which shows only one programmable solenoid valve was used to adjust the required airflow to 3 bending pMAs. This was a design choice as in the first place the researchers were interested to keep the system simple for the proof of concept. There are other reasons for having only one solenoid valve such as practical considerations and removing cross terms and interactions which in turn simply behaviour, its analysis and examination of the closed loop system.

For maintaining the required airflow the MATRIX 3/3 750 series solenoid valve with four ports has been chosen as the control actuator. This is a normally closed valve controlled by two 24V Pulse Width Modulation (PWM) signal from its control unit (shown by PWM in the figure and includes Arduino UNO from Mechatronics), which allows both vent or fill actively. A driver circuit provides an interface between the solenoid valve and the control unit. Additionally, the valve nature enables the pMAs to maintain its position without consuming control energy. As a matter of design only one pressure transducer sensor have been incorporated in the pneumatic circuit to monitor the internal state of the muscles and provide feedback to the control system. The complete unit can safely withstand pressures up to $1.2 \mathrm{MPa}$, although $500 \mathrm{kPa}$ (5 bar) is the operating pressure for the pneumatic system. The pressure sensor is connected directly to the pMAs and the controller. The output of the pressure sensor is in volt ranges between 0.5 to 4.5 VDC.

\section{B. Dynamical Modelling}

The schematic representation of the pneumatic system setup shown in Fig. 7 consists of three pMAs, which essentially have distributed parameter, stochastic, non-linear and time varying nature, convoluted further with dead zone, time delay and saturation due to non-linear characteristics of solenoid valve and pipes. The nonlinearity in pMAs subsystem can be modelled based on advanced geometric muscle model obtained earlier. The distributed parameters nature of pMA has been shown through Finite Element Analysis (FEA) by Irshaidat [20]. The stochastic and time varying nature is due to intrinsically pneumatic characteristic of the EpMAE and its prototype construction. Having above characteristics simultaneously present a challenge, not only in capturing them appropriately in modelling of the overall system dynamics, but also in developing suitable control strategy based on this model that can stabilize and regulate the system properly.

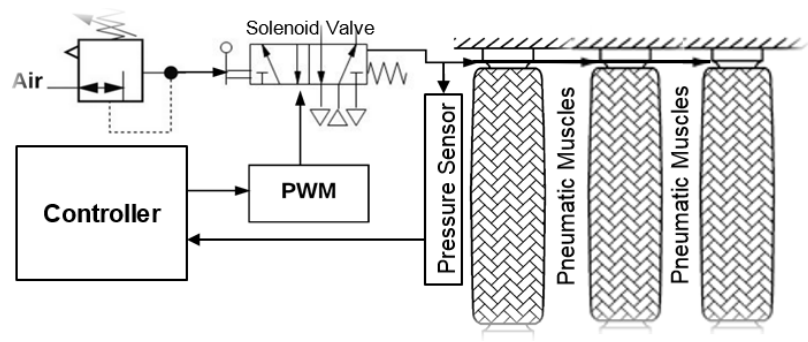

Figure 7. Pneumatic diagram of the experimental actuator 
Tackling above challenge is one of the novelty of this paper. To begin with this modelling challenge, here we have purposed that the core dynamics of the system can predominantly be simplified by visualising the electric circuit equivalent of the EpMAE. From this a linear timeinvariant model, say $\mathrm{Gp}^{*}(\mathrm{~s})$, can be constructed for the EpMAE prototype. This will consequently allow that any relevant characteristic such as nonlinearity and time delay can incrementally be added to the core dynamic model if it is required to satisfy control system design requirements.

\section{Model Validation}

Considering the equivalent electric circuit of the core dynamic of the system offers a simple and the same time powerful model structure that can provide a deeper insight and understanding of the nature of the system under the control. From experiment the parameters of this model have been obtain under certain lab conditions and consequently validated. Considerably important, each element in this model structure have the freedom to fluctuate by time, temperature and other system dependent or independent factors so that it can represent various nonlinear and time varying behaviour of the EpMAE prototype. Also as mentioned earlier, if required, additional interesting characteristics such as transport delay and dead-zone can easily be added to the model because of superposition principal. Especially this is true for a common scenario that core dynamic system exhibits different behaviour based on operating condition, i.e., filling or venting and we denote the transfer functions $\operatorname{Gpf}(\mathrm{s})$ and $\operatorname{Gpv}(\mathrm{s})$ refer to $\mathrm{Gp}^{*}(\mathrm{~s})$ for filling and venting operating condition respectively.

\section{Control System Design}

In order to make the EpMAE suitable to a wider stroke population apart from design requirements such as portability and lightweight, which has been discussed earlier, there are other requirements related to rehabilitation regimes, which have widely been studied by many researchers [2123]. From a system engineering point of view, apart from developing a pattern of repetitive motion according to specific rehabilitation regime, it also requires to develop an appropriate control strategy to satisfy above requirements. Most importantly the control strategy is required to follow specific practice profile within human ability with stroke, which can be captured by developing a suitable model. Therefore the main task of controller, apart from providing stability and dealing with time varying and nonlinear dynamic of the system, is to make EpMAE to follow this model as reference, hence suggesting Model Reference Adaptive Control (MRAC) strategy [24] to be used. Fig. 8 illustrates the MRAC that was developed for the EpMAE prototype. In this figure "r", "Ym", "Yp" "e", and "U", are referred to pressure set-point, the output of desired reference model M(s) and the output of the Plant $\mathrm{Gp}^{*}(\mathrm{~s})$, i.e., output EpMAE prototype in term of pressure, the error between reference model and the plant and the manipulated control variable calculated by the controller to provide right input to EpMAE..

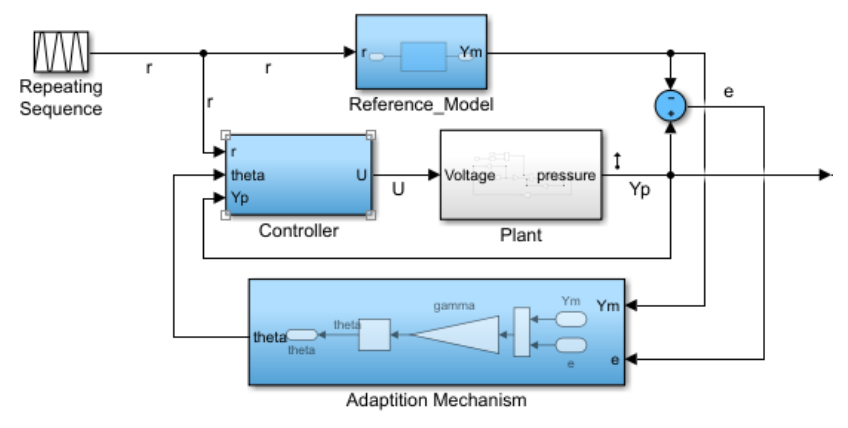

Figure 8. MRAC schematic, which was used to control the pressure inside EpMAE prototype.

In Fig. 8, the adaption mechanism "theta(s)" can be obtained by considering following relationship

$$
\operatorname{theta}(\mathrm{s})=-\frac{\text { gamma }}{\mathrm{s}}(\mathrm{Yp}-\mathrm{Ym}) \mathrm{Ym}
$$

Having higher the value of gamma will result in a higher sensitive to the plant changes or faster adaptation. The internal controller signal "u" will be adjusted and scaled by theta(s) to produce final manipulated control variable " $U$ ", i.e.,

$$
\mathrm{U}(\mathrm{s})=\mathrm{u}(\mathrm{s}) \operatorname{Theta}(\mathrm{s})
$$

It is expected that the control law that synthesised by above formula and based on dynamic characteristics of the EpMAE prototype $\mathrm{Gp}^{*}(\mathrm{~s})$, would met above rehabilitation requirement, i.e., to provide appropriate functional rehabilitation profiles effectively for long duration, bearable and high intensity usage of the device. However there is another obstacle to use this control strategy for the novel exoskeleton soft robotic arm since "U" only controls pressure and not the position or orientation of the robotic arm. In order to control robot arm orientation, it is necessary to obtain its invers dynamics, and then applied to the prototype. This is discussed in the next section.

\section{E. Control Implementation}

In order to implement the control law obtained in last section, the inverse and direct kinematics of the soft robotic arm obtained from analysis and experimental results in previous sections was implemented as lookup tables using Simulink as shown in Fig. 9.

The rehabilitation sequence is a repeating pattern so that the soft robotic arm angle $\theta$ increases from 0 degree to 150 degree slowly within 40 seconds, then after 20 seconds rest, it returns to initial value with another 20 seconds rest (see Fig. 9). The Fig. 10 shows also the obtained angle of the soft robotic arm, which follows the rehabilitation profile very well except on small angles due to the fact that the air pressure inside the soft robotic arm can never reaches to zero. 


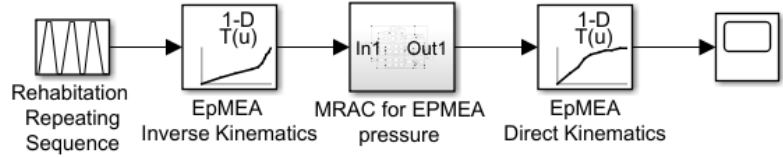

Figure 9. Implementation of control law, inverse and direct kinematics of the soft robotic arm EpMAE prototype

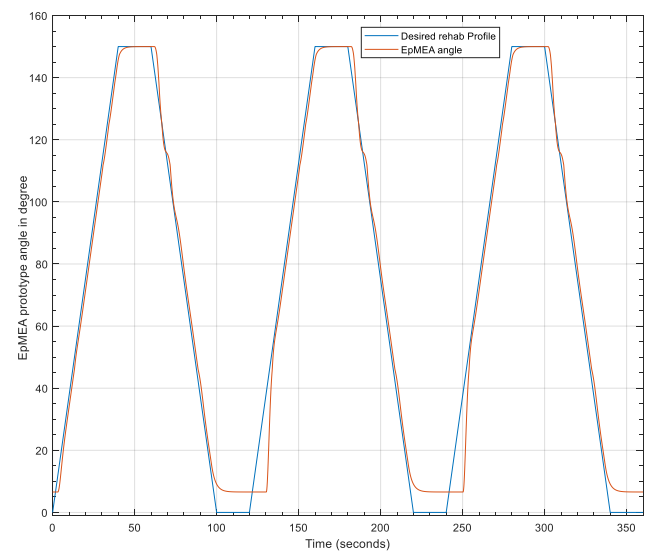

Figure 10. The rehabilitation sequence and the response of soft robotic arm EpMAE prototype in degree.

\section{CONCLUSION}

The goal of this paper is to introduce a soft robotic arm that can independently be used by individuals who suffer from lack of mobility due to stroke or other similar disabling illness. One of the major future work and aims in developing such a solution is to create a rehabilitation device that is portable, easy to use at home so that users can practice intensive rehabilitation without therapists at home in parallel with daily living activities.

A novel exoskeleton soft robotic arm, which is wearable, lightweight and portable, was introduced. The proposed arm consists of various innovative pneumatic Muscle Actuators capable of bending in contrary to traditional pMA. Experimental characterization showed how well this soft robotic arm meets the requirements. This with the proposed controller has produced very encouraging results for further works which include further characterization of the EpMAE using geometrical modelling and numerical finite element methods, use neural network to capture nonlinear dynamic and develop MRAC based on Neural Networks. This will then hopefully open doors for clinical trials and using the device with patients.

\section{REFERENCES}

[1] C.-Y. Huang, Y.-C. Li, H.-K. Wang, P.-S. Sung, L.-C. Wang, Y.-T. Sun, et al., "Stroke suggests increased risk of dementia," Current Alzheimer Research, vol. 12, pp. 287-295, 2015.

[2] S. Hamid and R. Hayek, "Role of electrical stimulation for rehabilitation and regeneration after spinal cord injury: an overview," European Spine Journal, vol. 17, pp. 1256-1269, 2008.

[3] G. Kwakkel, B. J. Kollen, J. van der Grond, and A. J. Prevo, "Probability of regaining dexterity in the flaccid upper limb: impact of severity of paresis and time since onset in acute stroke," Stroke, vol. 34, pp. 2181-2186, 2003.

M. A. M. Dzahir and S.-i. Yamamoto, "Recent trends in lowerlimb robotic rehabilitation orthosis: Control scheme and strategy for pneumatic muscle actuated gait trainers," Robotics, vol. 3, pp. 120-148, 2014.

H. I. Krebs, N. Hogan, M. L. Aisen, and B. T. Volpe, "Robotaided neurorehabilitation," IEEE transactions on rehabilitation engineering, vol. 6, pp. 75-87, 1998.

P. S. Lum, C. G. Burgar, M. Van der Loos, P. C. Shor, M. Majmundar, and R. Yap, "MIME robotic device for upper-limb neurorehabilitation in subacute stroke subjects: A follow-up study," Journal of rehabilitation research \& development, vol. 43, pp. 631-643, 2006.

R. Loureiro, F. Amirabdollahian, M. Topping, B. Driessen, and W. Harwin, "Upper limb robot mediated stroke therapyGENTLE/s approach," Autonomous Robots, vol. 15, pp. 35-51, 2003.

M. Curtin, M. Molineux, and J.-A. Webb, Occupational Therapy and Physical Dysfunction E-Book: Enabling Occupation: Elsevier Health Sciences, 2009.

K. Kiguchi, R. Esaki, T. Tsuruta, K. Watanabe, and T. Fukuda, "An exoskeleton system for elbow joint motion rehabilitation," in Advanced Intelligent Mechatronics, 2003. AIM 2003. Proceedings. 2003 IEEE/ASME International Conference on, 2003, pp. 1228-1233.

J. S. Sulzer, M. A. Peshkin, and J. L. Patton, "Design of a mobile, inexpensive device for upper extremity rehabilitation at home," in Rehabilitation Robotics, 2007. ICORR 2007. IEEE 10th International Conference on, 2007, pp. 933-937.

J. Rosen, M. Brand, M. B. Fuchs, and M. Arcan, "A myosignalbased powered exoskeleton system," IEEE Transactions on systems, Man, and Cybernetics-part A: Systems and humans, vol. 31, pp. 210-222, 2001.

R. Song, K.-y. Tong, X. Hu, and L. Li, "Assistive control system using continuous myoelectric signal in robot-aided arm training for patients after stroke," IEEE transactions on neural systems and rehabilitation engineering, vol. 16, pp. 371-379, 2008.

K. Kiguchi, K. Iwami, M. Yasuda, K. Watanabe, and T. Fukuda, "An exoskeletal robot for human shoulder joint motion assist," IEEE/ASME transactions on mechatronics, vol. 8, pp. 125-135, 2003.

G. Fazekas, M. Horvath, T. Troznai, and A. Toth, "Robotmediated upper limb physiotherapy for patients with spastic hemiparesis: a preliminary study," Journal of rehabilitation medicine, vol. 39, pp. 580-582, 2007.

A. Al-Ibadi, S. Nefti-Meziani, and S. Davis, "Efficient structurebased models for the McKibben contraction pneumatic muscle actuator: the full description of the behaviour of the contraction PMA," in Actuators, 2017, p. 32.

S. Davis and D. G. Caldwell, "Braid effects on contractile range and friction modeling in pneumatic muscle actuators," The International Journal of Robotics Research, vol. 25, pp. 359$369,2006$.

A. Al-Ibadi, S. Nefti-Meziani, and S. Davis, "Active soft end effectors for efficient grasping and safe handling," IEEE Access, vol. 6, pp. 23591-23601, 2018.

H. Al-Fahaam, S. Davis, and S. Nefti-Meziani, "The design and mathematical modelling of novel extensor bending pneumatic artificial muscles (EBPAMs) for soft exoskeletons," Robotics and Autonomous Systems, vol. 99, pp. 63-74, 2018.

D. A. Winter, Biomechanics and motor control of human movement: John Wiley \& Sons, 2009.

M. Irshaidat, "Design and Implementation of a Novel Lightweight Soft Upper Limb Exoskeleton Using Pneumatic Actuator Muscles," PhD, Salford University, 2018.

P. Langhorne, J. Bernhardt, and G. Kwakkel, "Stroke rehabilitation," The Lancet, vol. 377, pp. 1693-1702, 2011.

M. M. Pinter and M. Brainin, "Rehabilitation after stroke in older people," Maturitas, vol. 71, pp. 104-108, 2012.

E. Ernst, "A review of stroke rehabilitation and physiotherapy," Stroke, vol. 21, pp. 1081-1085, 1990.

J. Sun, "Model Reference Adaptive Control," Encyclopedia of Systems and Control, pp. 729-734, 2015. 